\title{
Foot trajectory approximation using the pendulum model of walking
}

\author{
Juan Fang • Kenneth J. Hunt
}

Received: 8 April 2013/Accepted: 5 September 2013/Published online: 21 September 2013

(C) International Federation for Medical and Biological Engineering 2013

\begin{abstract}
Generating a natural foot trajectory is an important objective in robotic systems for rehabilitation of walking. Human walking has pendular properties, so the pendulum model of walking has been used in bipedal robots which produce rhythmic gait patterns. Whether natural foot trajectories can be produced by the pendulum model needs to be addressed as a first step towards applying the pendulum concept in gait orthosis design. This study investigated circle approximation of the foot trajectories, with focus on the geometry of the pendulum model of walking. Three able-bodied subjects walked overground at various speeds, and foot trajectories relative to the hip were analysed. Four circle approximation approaches were developed, and best-fit circle algorithms were derived to fit the trajectories of the ankle, heel and toe. The study confirmed that the ankle and heel trajectories during stance and the toe trajectory in both the stance and the swing phases during walking at various speeds could be well modelled by a rigid pendulum. All the pendulum models were centred around the hip with pendular lengths approximately equal to the segment distances from the hip. This observation provides a new approach for using the pendulum model of walking in gait orthosis design.
\end{abstract}

Keywords Pendulum model · Gait - Locomotion . Ankle trajectory $\cdot$ Toe trajectory $\cdot$ Rehabilitation robotics

\footnotetext{
J. Fang $\cdot$ K. J. Hunt $(\bowtie)$

Division of Mechanical Engineering, Department of Engineering and Information Technology, Institute for Rehabilitation and Performance Technology, Bern University of Applied Sciences, 3400 Burgdorf, Switzerland

e-mail: Kenneth.hunt@bfh.ch
}

\section{Introduction}

Generating a natural foot trajectory is a primary focus for design of gait orthoses. Task-specific training paradigms suggest methods for practical implementation of walking-like exercise for gait restoration [13]. Rehabilitation robots for walking aim to move the thigh, shank and foot in a walking-like manner. Some gait robots adopt end-effector control which moves the foot along the target trajectory so as to achieve a synchronized walking pattern in the lower limbs during locomotion training $[4,6,7,10,14]$.

Human locomotion in the stance phase is often described by an inverted pendulum, but the application of the pendulum model in bipedal walkers adopts an inconsistent pendular length. The human body's centre of mass rises and falls in an arc shape during each stride, which leads to a pendulum description of the stance phase $[1,2]$. The leg stands straight on the ground and progresses like a rigid bar pivoting on the foot. This resembles the movement of an inverted pendulum, which has a pendular length equal to the leg length [18]. Based on the rocker function of the heel, ankle and forefoot [22], a roll-over-shaped foot is often used to assist smooth progression in the application of the pendulum model in the design of bipedal walkers [19]. However, in order to obtain a reasonably anthropometric foot shape, most semicircular feet have radii shorter than the shank length $[12,19]$. In this application, the pendulum model has a different length from the initial observation of the inverted pendulum length equal to that of the leg. To apply the inverted pendulum model to the design of a gait orthosis focusing on leg kinematics, it is necessary to determine an optimal pendular length to generate the stance foot trajectory occurring during normal gait. 
Similar to the stance leg, the swing leg also has a pendulum-like property [18], which has been exemplified in passive walkers [20]. Provided with an appropriate initial speed and position, the leg, without much internal muscle work, achieves the swing phase by passive interaction with gravity [21]. The pendulum characteristics of ballistic walking inspired the development of bipedal passive walkers [20]. The walker has two rigid legs linked together by a frictionless pin joint at the hip. One leg rides on the semicircular foot, and simultaneously, the other leg swings forwards in a pendular arc, resulting in rhythmical gait patterns $[17,20]$. The bipedal walker has been modified for potential application in rehabilitation technology. It generally includes an actuated knee joint for toe clearance and additional control mechanisms for stability [3, 17, 25], which results in a complicated robotic system. It is of interest to investigate whether the simple rigid pendulum model can be applied in the design of robotic gait orthoses. As gait orthoses are generally intended to duplicate the leg kinematics of normal gait, it is worthwhile investigating whether the pendulum model of walking simulates well the trajectory of a swinging foot.

The aim of this feasibility study was to investigate whether foot trajectories can be generated by a pendulum model. Approximation algorithms were developed to find the best-fit circles of foot trajectories relative to the hip, with focus on the ankle, heel and toe. Fang et al. [9] investigated circle approximation of the toe trajectory. The present work expands the approximation algorithms and finds the best-fit circles for the ankle, heel and toe trajectories at various walking speeds.

\section{Methods}

\subsection{Gait analysis experiment}

A gait experiment was performed using a Vicon motion analysis system (Oxford Metrics Ltd., Oxford, UK). Ethical approval was obtained from the Departmental Ethics Committee at the University of Strathclyde, Glasgow, UK. Consistent with the feasibility study design, three representative able-bodied subjects were recruited (Table 1) and they provided written informed consent prior to participation. The Vicon Clinical Manager marker set was used to record the lower limb trajectories with a sampling frequency of $120 \mathrm{~Hz}$ [15]. The gait experiment started with a static trial for estimation of the joint centres and axes. The subjects were then asked to walk barefoot along a $10-\mathrm{m}$ walkway at 100, 75 and $50 \%$ of their normal cadence. Tests at each speed were repeated three times.

The analysis software BodyBuilder (Oxford Metrics Ltd., Oxford, UK) was used to reconstruct the foot
Table 1 Subject information

\begin{tabular}{lllll}
\hline Subject & Gender & Age (years) & Mass $(\mathrm{kg})$ & Leg length $(\mathrm{m})$ \\
\hline S1 & Male & 24 & 94.7 & 1.00 \\
S2 & Female & 42 & 74.4 & 0.91 \\
S3 & Female & 33 & 67.8 & 0.88 \\
\hline
\end{tabular}

segments and to estimate the trajectories. A reference system was defined with centre $(0,0)$ at the hip joint (Fig. 1). Trajectories of the ankle, heel and toe in this reference system were analysed.

\subsection{Circle approximation algorithms}

The pendulum setup was determined by finding the approximated circles for the ankle, heel and toe trajectories. It was assumed that each foot segment analysed had $Q$ data points $\left(x_{i}, y_{i}\right)(i=1,2,3 \ldots \mathrm{Q})$. The best-fit circle can be determined by solving the total least squares problem [5]:

$$
\begin{aligned}
f\left(x_{i}, y_{i}\right)= & \sum_{i=1}^{Q}\left(\sqrt{\left(x_{i}-x_{c}\right)^{2}+\left(y_{i}-y_{c}\right)^{2}}-r\right)^{2} ; \\
& (i=1,2,3 \ldots Q) .
\end{aligned}
$$

Fig. 1 Foot segment distances. Black dots at the lateral malleolus, calcaneus and second metatarsal head indicate the ankle, heel and toe, respectively. The distances from the hip centre to the ankle, heel and toe are represented by dashed lines as $l_{A}, l_{H}$ and $l_{T}$, respectively

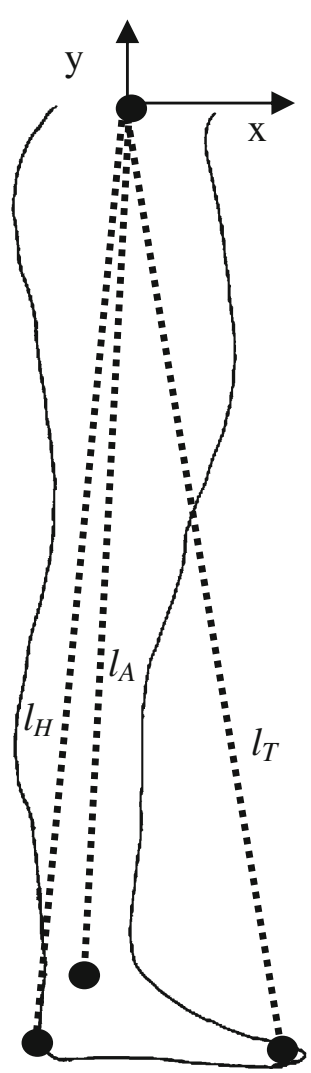


where $\left(x_{c}, y_{c}\right)$ is the fit circle centre and $\mathrm{r}$ is the circle radius. However, this is a nonlinear problem, so a modified least squares criterion $F\left(x_{i}, y_{i}\right)$ was used here [16]:

$$
\begin{gathered}
F\left(x_{i}, y_{i}\right)=\sum_{i=1}^{Q}\left(\left(x_{i}-x_{c}\right)^{2}+\left(y_{i}-y_{c}\right)^{2}-r^{2}\right)^{2} ; \\
(i=1,2,3 \ldots Q) .
\end{gathered}
$$

The values of $x_{c}, y_{c}$ and $r$ which satisfy $\min _{x_{c}, y_{c}, r} F\left(x_{i}, y_{i}\right)$ apply to $\min _{x_{c}, y_{c}, r} f\left(x_{i}, y_{i}\right)$, if the analysed data exactly match a circle. However, Eq. (2) was proven to be preferable to Eq. (1) in terms of robustness in the presence of outliers [5]. Therefore, this study searched for the values of $x_{c}, y_{c}$ and $r$ satisfying $\min _{x_{c}, y_{c}, r} F\left(x_{i}, y_{i}\right)$.

The first derivative of Eq. (2) over $r, x_{c}$ and $y_{c}$ yields:

$\mathrm{d}\left(F\left(x_{i}, y_{i}\right)\right) / \mathrm{d} r=0$

$\mathrm{d}\left(F\left(x_{i}, y_{i}\right)\right) / \mathrm{d} x_{c}=0$

$\mathrm{d}\left(F\left(x_{i}, y_{i}\right)\right) / \mathrm{d} y_{c}=0$

It was hypothesized that the centre of the fit circle $\left(x_{c}\right.$, $\left.y_{c}\right)$ was near to the hip joint $(0,0)$ and that its radius $r$ was approximately equal to the segment distance $l$ measured from the hip joint $\left[l\right.$ refers, respectively, to $l_{A}, l_{H}$ and $l_{T}$ for the ankle, heel and toe (Fig. 1)]. Four methods were developed to search for the fit circles:

1. For the $y$ coordinate of the circle at the hip $\left(y_{c}=0\right)$, search for the optimal $x_{c}$ and $r$. Equations (3)-(4) yield:

$$
\begin{aligned}
& -4 r \sum_{i=1}^{Q}\left[\left(x_{i}-x_{c}\right)^{2}+y_{i}^{2}-r^{2}\right]=0 \\
& -4 \sum_{i=1}^{Q}\left(x_{i}-x_{c}\right)\left[\left(x_{i}-x_{c}\right)^{2}+y_{i}^{2}-r^{2}\right]=0
\end{aligned}
$$

2. For the $x$ coordinate of the circle at the hip $\left(x_{c}=0\right)$, search for the optimal $y_{c}$ and $r$. Equations (3) and (5) yield:

$$
\begin{aligned}
& -4 r \sum_{i=1}^{Q}\left[x_{i}^{2}+\left(y_{i}-y_{c}\right)^{2}-r^{2}\right]=0 \\
& -4 \sum_{i=1}^{Q}\left(y_{i}-y_{c}\right)\left[x_{i}^{2}+\left(y_{i}-y_{c}\right)^{2}-r^{2}\right]=0
\end{aligned}
$$

3. For both the $x$ and $y$ coordinates of the circle at the hip $\left(x_{c}=0, y_{c}=0\right)$, search for the optimal radius $r$ :

$$
-4 r \sum_{i=1}^{Q}\left[x_{i}^{2}+y_{i}^{2}-r^{2}\right]=0
$$

4. For the radius $r$ equal to the segment distance, search for the optimal $x_{c}$ and $y_{c}$ :

$$
\begin{aligned}
& -4 \sum_{i=1}^{Q}\left(x_{i}-x_{c}\right)\left[\left(x_{i}-x_{c}\right)^{2}+\left(y_{i}-y_{c}\right)^{2}-l^{2}\right]=0 \\
& -4 \sum_{i=1}^{Q}\left(y_{i}-y_{c}\right)\left[\left(x_{i}-x_{c}\right)^{2}+\left(y_{i}-y_{c}\right)^{2}-l^{2}\right]=0
\end{aligned}
$$

These four methods were applied to the trajectories of the ankle, heel and toe separately. Solutions of Eqs. (6)(12) gave four fit circles for each segment trajectory.

\subsection{Comparison of methods}

The four methods were compared using the mean approximation error

$$
\begin{aligned}
E= & \frac{1}{Q} \sum_{i=1}^{Q}\left|\sqrt{\left(x_{i}-x_{c}\right)^{2}+\left(y_{i}-y_{c}\right)^{2}}-r\right| ; \\
& (i=1,2,3 \ldots Q) .
\end{aligned}
$$

The best-fit circle algorithm was the method with the smallest mean error [Eq. (13)]. The errors and other results related to length are presented as normalized values (\% of leg length).

\section{Results}

The three walking cadences resulted in a speed range of $0.43-1.44 \mathrm{~m} / \mathrm{s}$ (Table 2). The ankle and heel had a curved trajectory during the stance phase, while the toe trajectory had similarly-curved stance and swing phases (Fig. 2). Therefore, the ankle and heel trajectories in the stance phase and the toe trajectory over the whole gait cycle were considered for circle approximation.

The four approximation methods yielded four fit circles for each of the three foot segments (Fig. 2-S1: the other two subjects gave qualitatively similar results). All of the methods approximated the foot trajectories of the three subjects fairly well, with errors less than $3 \%$ (Table 3 ). Method 1, which fixed $y_{c}$ at the hip and allowed $x_{c}$ and $r$ to vary, had the smallest error and was determined as the bestfit circle algorithm forthwith.

The foot trajectories were fit closely by the circles obtained from the best-fit algorithms, with centres close to

Table 2 Walking speeds

\begin{tabular}{llll}
\hline Subjects & S1 & S2 & S3 \\
\hline Normal cadence (steps/min) & 107 & 93 & 112 \\
$100 \%$ of normal cadence $(\mathrm{m} / \mathrm{s})$ & 1.42 & 1.16 & 1.44 \\
$75 \%$ of normal cadence $(\mathrm{m} / \mathrm{s})$ & 0.81 & 0.79 & 0.95 \\
$50 \%$ of normal cadence $(\mathrm{m} / \mathrm{s})$ & 0.43 & 0.528 & 0.61 \\
\hline
\end{tabular}




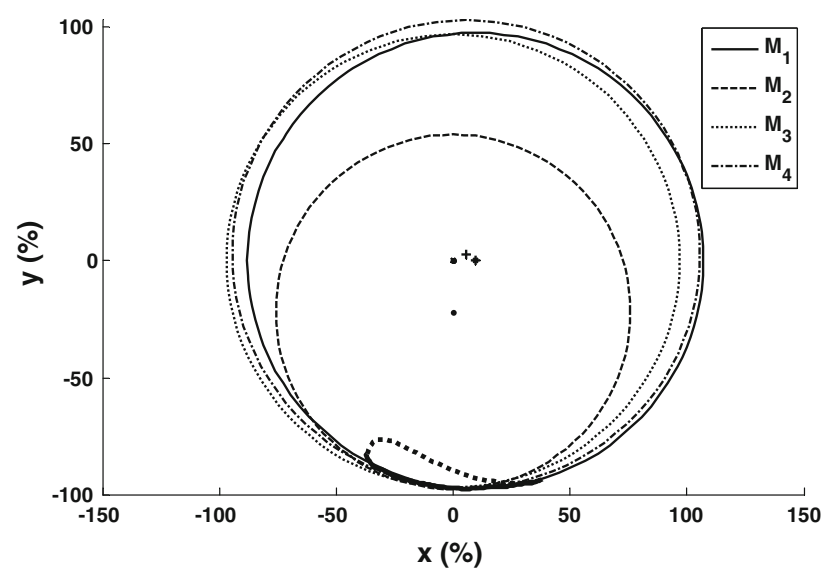

(a) Ankle

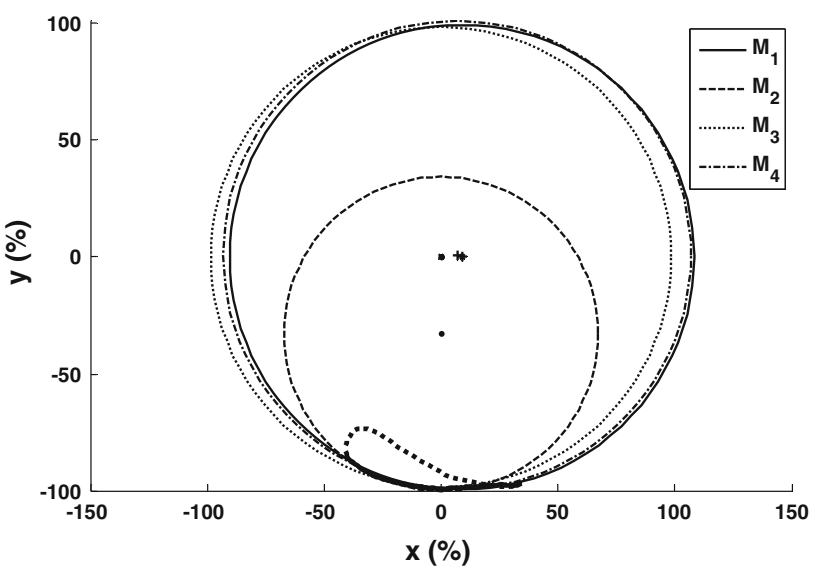

(b) Heel

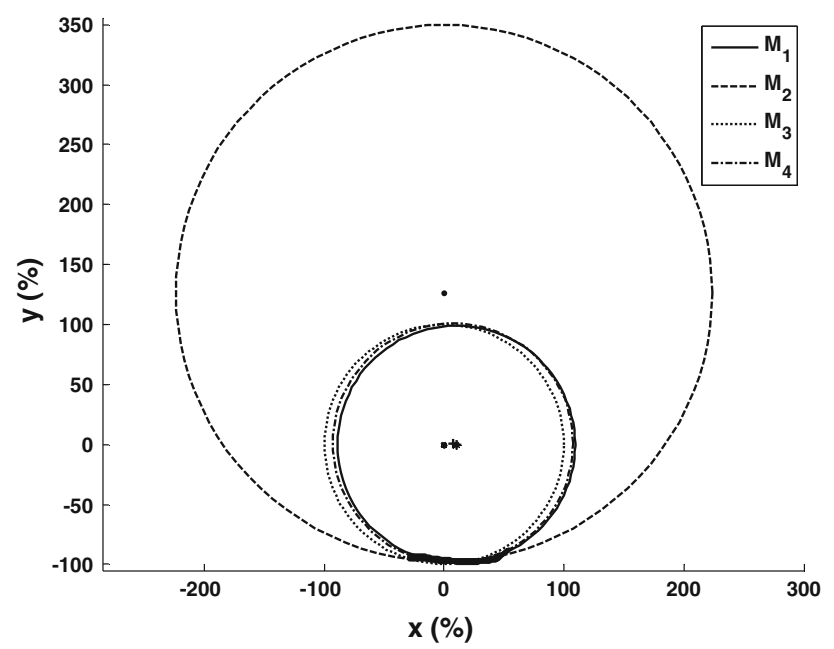

(c) Toe

the hip and radii almost equal to the segment distances from the hip (Figs. 3, 4). The errors between the best-fit circles and their respective ankle and heel trajectories during the stance phase (60\% of gait cycle) were less
Fig. 2 Circle approximation of the foot trajectories of S1 walking at normal cadence. Methods 1-4 ( $\mathrm{M}_{1}, \mathrm{M}_{2}, \mathrm{M}_{3}$ and $\left.\mathrm{M}_{4}\right)$ yielded fit circles as solid, dashed, dotted and dash-dot lines with the centres as stars, dots, crosses and plus signs, respectively. The foot trajectories considered for circle approximation are shown as thick solid lines, while those not involved in the approximation (the ankle and heel trajectories during swing) are thick dotted lines

Table 3 The errors (\%) from the four methods applied to the ankle, heel and toe trajectories in the three subjects walking at normal cadence

\begin{tabular}{llllll}
\hline & & Method 1 & Method 2 & Method 3 & Method 4 \\
\hline S1 & Ankle & 0.42 & 1.68 & 1.74 & 0.65 \\
& Heel & 0.77 & 1.31 & 1.50 & 0.12 \\
& Toe & 1.21 & 1.53 & 2.69 & 1.76 \\
S2 & Ankle & 0.52 & 1.01 & 1.13 & 0.93 \\
& Heel & 0.82 & 0.91 & 1.02 & 0.99 \\
& Toe & 1.44 & 1.61 & 2.43 & 1.74 \\
S3 & Ankle & 0.59 & 0.63 & 1.10 & 0.83 \\
& Heel & 1.05 & 1.24 & 1.29 & 1.17 \\
& Toe & 1.56 & 1.84 & 2.10 & 1.91 \\
\multirow{2}{*}{ Mean \pm SD } & $0.93 \pm 0.41$ & $1.31 \pm 0.40$ & $1.67 \pm 0.61$ & $1.12 \pm 0.59$ \\
\hline
\end{tabular}

$S D$ standard deviation
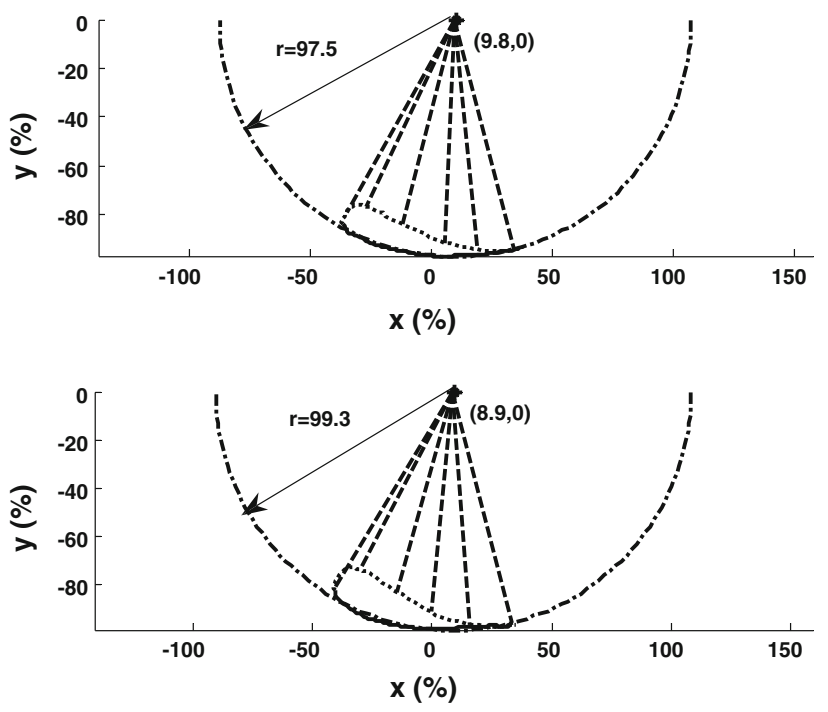

Fig. 3 Best-fit circles of the ankle (upper) and heel (lower) trajectories in the stance phase of S1 walking at normal cadence. The ankle and heel trajectories in the stance phase are shown as solid lines. The corresponding best-fit circles are shown as dash-dot lines, with the centre given in brackets and the radius represented as an arrow. The trajectories in the swing phase, which are not involved in the approximation, are dotted lines. The dashed lines show the distance between the trajectories during swing and their fit-circle centres

than $1.5 \%$ (Fig. 5). The toe trajectory during both the stance and swing phases is quite well approximated by its best-fit circle (Fig. 4), with a maximal error of about $4 \%$ (Fig. 5). 

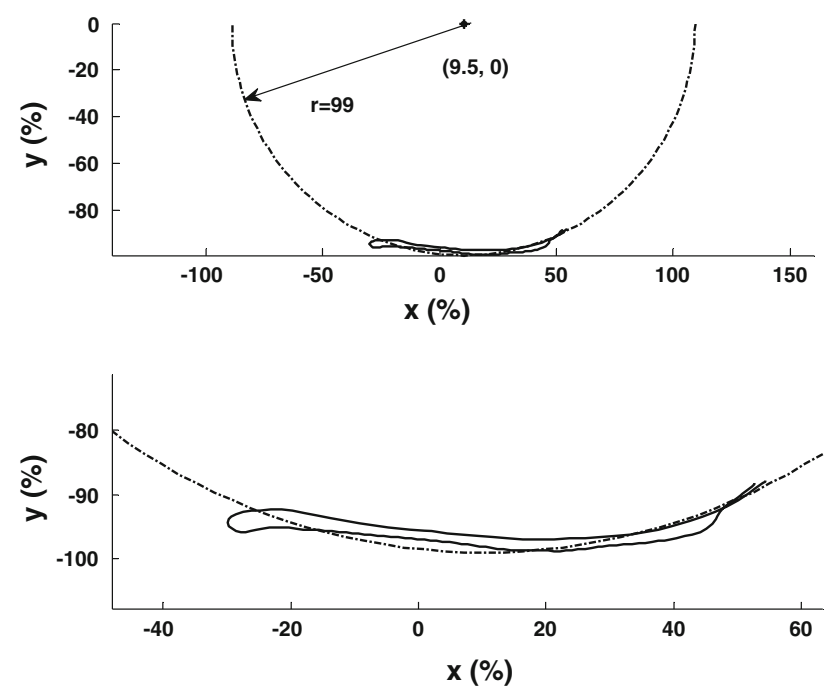

Fig. 4 Best-fit circle (dash-dot lines) of the toe trajectory (solid lines) over the whole gait cycle of S1 walking at normal cadence. Upper The circle has the centre given in brackets and the radius represented as an arrow. Lower A zoomed view

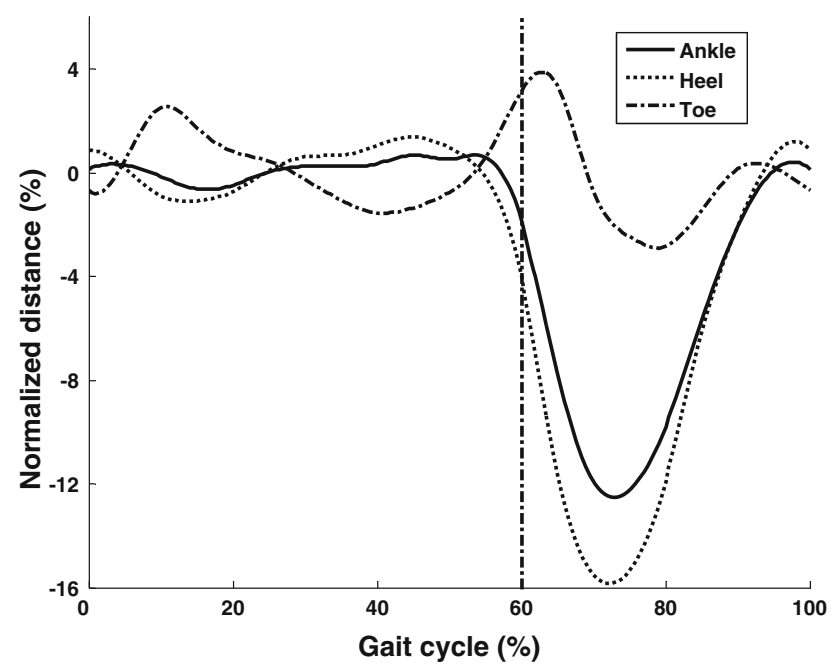

Fig. 5 The distances between the best-fit circle and the foot segments over the whole gait cycle of S1 walking at normal cadence (the vertical dash-dot line divides the whole cycle into stance and swing phases)

The foot trajectories during slower walking were also curved. The ankle trajectories in the stance phase (Fig. 6) at different speeds were best fitted by circles of similar radii. This also applies to the toe trajectories over the whole gait cycle at different speeds (Fig. 7). When the speed decreases, the foot trajectories are shortened, with the trajectory behind the hip joint (the negative part) reduced faster than that before the hip joint (the positive part). Therefore, the best-fit circle centre moves further from the hip joint centre at slower speeds. Applying Method 1 to the ankle, heel and toe trajectories

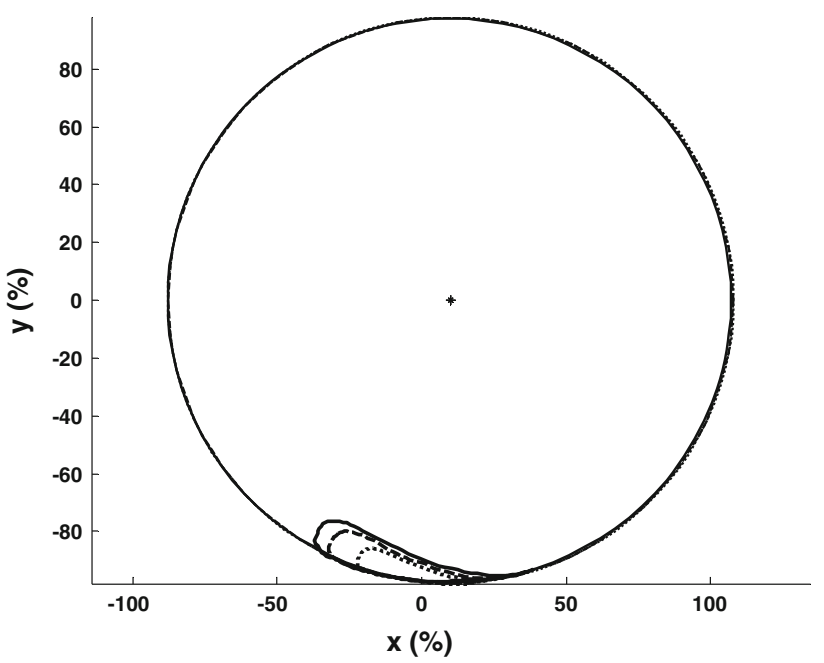

Fig. 6 The best-fit circles of the ankle trajectories during stance in S1 walking at $100 \%$ (solid line), $75 \%$ (dashed line) and $50 \%$ (dotted line) of normal cadence
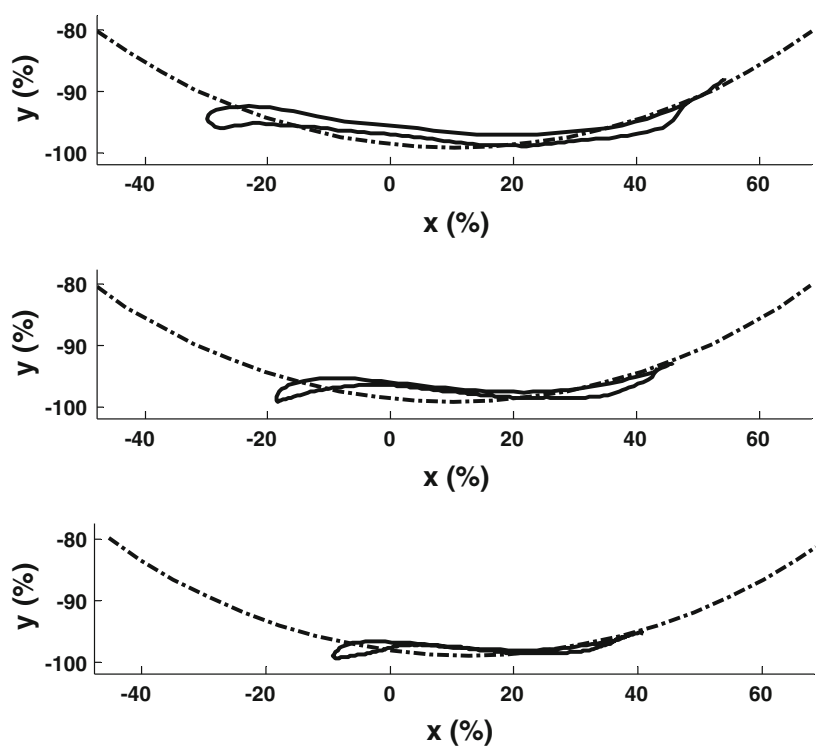

Fig. 7 The best-fit circles (dash-dot curve) of the toe trajectories (solid lines) over the whole gait cycle in S1 walking at $100 \%$ (top), $75 \%$ (middle) and $50 \%$ (bottom) of normal cadence

at three different speeds for all subjects yielded the geometries of their respective best-fit circles (Table 4). The foot trajectories at $100 \%$ of normal cadence had best-fit circles centred nearest to the hip, while those at $50 \%$ of normal cadence had radii closest to the segment distances (Table 4). The best-fit circle approximation for all the foot trajectories had a mean error less than $2 \%$, with the smallest error in the ankle trajectory during stance (Fig. 8). When the speed decreased, the error reduced. 
Table 4 Mean centres $x_{c}$ and radii $r$ of best-fit circles (\%) for the ankle, heel and toe trajectories in the three subjects walking at various speeds

\begin{tabular}{|c|c|c|c|c|c|c|c|}
\hline \multirow[t]{2}{*}{ Subject } & \multirow[t]{2}{*}{ Segment } & \multicolumn{2}{|c|}{$100 \%$ of cadence } & \multicolumn{2}{|c|}{$75 \%$ of cadence } & \multicolumn{2}{|c|}{$50 \%$ of cadence } \\
\hline & & $x_{c}$ & $r$ & $x_{c}$ & $r$ & $x_{c}$ & $r$ \\
\hline \multirow[t]{3}{*}{$\mathrm{S} 1$} & Ankle & 8.93 & 97.47 & 10.36 & 97.85 & 11.05 & 97.79 \\
\hline & Heel & 8.43 & 99.26 & 9.73 & 99.77 & 11.52 & 99.90 \\
\hline & Toe & 9.61 & 99.15 & 10.08 & 99.21 & 10.96 & 99.06 \\
\hline \multirow[t]{3}{*}{$\mathrm{S} 2$} & Ankle & 5.67 & 94.18 & 5.49 & 94.31 & 7.84 & 94.36 \\
\hline & Heel & 4.71 & 97.11 & 4.95 & 97.24 & 7.78 & 97.48 \\
\hline & Toe & 5.74 & 97.74 & 4.16 & 98.29 & 4.99 & 98.18 \\
\hline \multirow[t]{3}{*}{$\mathrm{S} 3$} & Ankle & 3.78 & 96.36 & 5.16 & 97.00 & 4.93 & 96.71 \\
\hline & Heel & 3.24 & 98.98 & 5.11 & 99.71 & 4.86 & 99.37 \\
\hline & Toe & 5.16 & 99.68 & 2.57 & 100.13 & 3.82 & 99.91 \\
\hline \multicolumn{2}{|c|}{ Mean $\pm \mathrm{SD}$} & $6.14 \pm 2.30$ & $97.77 \pm 1.76$ & $6.40 \pm 2.88$ & $98.17 \pm 1.84$ & $7.53 \pm 3.05$ & $98.08 \pm 1.78$ \\
\hline
\end{tabular}

$S D$ standard deviation

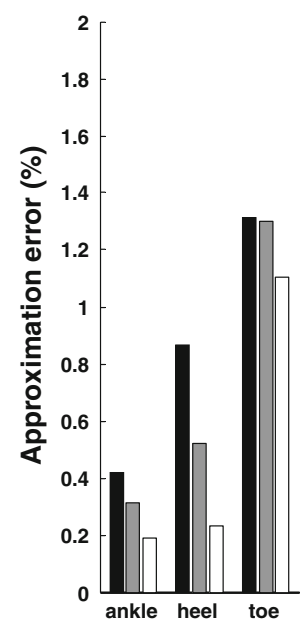

(a) S1

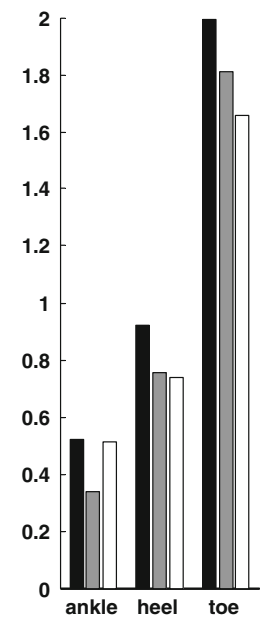

(b) S2

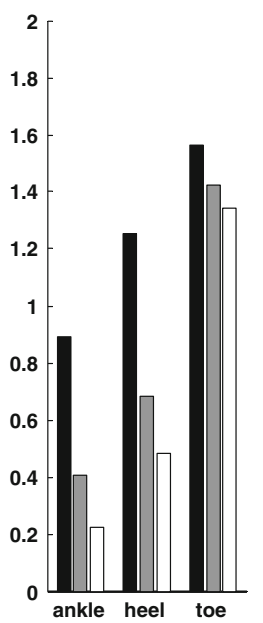

(c) $\mathrm{S} 3$

Fig. 8 Mean errors from the best-fit circle approximation (Method 1) for the three subjects walking at $100 \%$ (black), $75 \%$ (grey) and $50 \%$ (white) of normal cadence, respectively

\section{Discussion}

The aim of this feasibility study was to investigate whether foot trajectories can be generated by a pendulum model. Using adapted best-fit circle approximation algorithms, this study derived the pendulum model with optimal geometry to describe the foot trajectories. The ankle and heel moved along a circular path relative to the hip joint during the stance phase, while the toe trajectory over the whole gait cycle was described by a pendulum. All the pendulum models were based around the hip with pendular lengths approximately equal to the segment distances from the hip.

The foot trajectories during stance were well approximated by circles, which supports the concept of a roll-overshaped foot described previously [12, 19]. Hansen et al. [12] observed an arc shape of the trajectory of the centre of

pressure in stance and investigated the arc radius using a circle-fit approach, while the present work analysed the foot trajectories over the whole gait cycle with generalized best-fit circle algorithms. Furthermore, this study provided detailed information on the pendulum geometries for the ankle, heel and toe trajectories at various speeds, with a new contribution in fitting the toe trajectory over the whole gait cycle.

This study employed four circle approximation approaches, which found well-fit circles for the foot trajectories. This validates the hypothesis that the foot segment trajectories can be fit by circles centred near to the hip with radii approximately equal to the segment distances. Method 3 obtained a circle fitting the trajectory fairly well, which provided the basis for adopting the pendulum model of walking with its centre at the hip and radius equal to the leg length, as employed previously $[2,21]$. Introducing the hip as the base of the pendulum was an intuitive choice, but neither the ankle, heel nor toe trajectories were equally divided by the vertical line from the hip joint (Fig. 2). Therefore, Methods 1, 2 and 4 which allowed variation of the pendulum base around the hip outperformed Method 3 which assumed a fixed circle centre at the hip. Method 2 yielded fit circles with radii much larger or much smaller than the segment distances (Fig. 2). Therefore, it is feasible to set up a pendulum model of walking with a length longer than the leg [11] or a much shorter length [12, 19].

The foot segment trajectories were fit best with Method 1, which allowed horizontal adjustment of the pendulum base. The ankle trajectory had a smaller error from its bestfit circle than the heel or toe. This is probably because the only joint (the knee) between the ankle and the hip is almost in constant extension, making the thigh and shank behave as a rigid pendulum, while the heel and toe have slightly rotating joints, such as the ankle and subtalar joints [24]. During the swing phase, the knee is flexed, which 
shortens the distance from the hip to the ankle joint and the heel (Fig. 5). However, foot rotation lowers the toe, which results in a negligible change in the total distance between the hip and the toe. Therefore, the toe trajectory over the whole gait cycle was approximated by a circle. This observation does not mean that the foot will scuff the floor. It is reported that during the swing phase, the trunk rises about 4-5 cm [11], therefore, the whole swing leg is lifted off the ground. It is also reported that a swing leg during normal gait produces a minimal toe clearance of $5 \mathrm{~cm} \mathrm{[23].}$ This confirmed that there is a limited change in the distance between the toe and the hip during locomotion, which justifies the approach in this study to approximate the toe trajectory over the whole gait cycle with a circle. The pendulum successfully models the foot trajectories during locomotion at variable speeds, with negligible variations in the pendulum setup.

Determination of the pendulum geometry provides a new design approach for walking robots and lower limb orthoses. The general application of the pendulum structures in rehabilitation is seen in bipedal walkers with an actuated knee joint, which exploits gravity to achieve rhythmic gait patterns [3]. Much attention has been paid to the stability of the system, while the foot trajectories have not been specifically investigated. The pendulum model in this study lends itself to straightforward implementation using a rigid driven bar for accurate toe movement. Based on this, a gait orthosis can be developed, which includes a swaying rigid bar to guide the toe movement and a lengthadjustable bar (length change profile as in the solid line in Fig. 5) to achieve ankle rotation. As the best-fit circles of the toe trajectories at different speeds have a negligible variability in the circle radius (Table 4), the rigid bar for the toe trajectories can have a fixed length, i.e. approximately equal to the user's leg length. Since the best-fit circle centre ( $x_{c}$ in Table 4) moves further from the hip when the speed reduces, the pivot of the rigid bar can be adjusted to be further from the hip joint to produce the toe trajectories at slower speeds. Based on the end-effector principle [14], a synchronized walking pattern in the lower limbs can be achieved when the foot (the ankle and toe) moves along the target trajectories of normal gait. In contrast to actuated bipedal passive walkers, gait orthoses based on the rigid pendulum model can produce accurate foot movement, as long as the distance between the ankle and the hip is actuated as according to the solid line in Fig. 5. Compared to a general gait orthosis which controls the leg through direct actuation of the hip, knee and ankle joints, the proposed gait orthosis can achieve the target motion of each leg using only two actuators: one for toe movement and the other for ankle rotation. As the gait orthosis based on this approach might have a risk of foot scuffing during overground walking, for example, if there is an obstruction of $5 \mathrm{~cm}$ in height on the ground, we successfully implemented this new approach in a rehabilitation gait orthoses for supine stepping, where there is no floor to interrupt the foot movement $[8,9]$.

A limitation of this study was that only three points on the foot (the ankle, heel and toe) were investigated as the potential tips of the pendulum. There might be other points on the foot moving in circular paths.

The number of subjects recruited, three, is consistent with the feasibility design of the study but further research using data from more subjects is required to generalize the results. The three subjects recruited in the gait analysis experiment were quite broadly representative of the ablebodied population: they had a wide range of body mass (68-95 kg) and leg length $(0.88-1.00 \mathrm{~m})$, and they repeated their walking three times at a wide range of speeds $(0.43-1.44 \mathrm{~m} / \mathrm{s})$. After comparing the gait patterns with the literature on normal gait [24], we believe the recruited subjects provided unequivocal and consistent data representing the general gait pattern for healthy people. Therefore, the major observations, including (a) the circular trajectories of the ankle and heel during the stance phase and (b) the circular trajectory of the toe during the whole gait cycle, are consequences of the inherent kinematics of human walking and should be broadly applicable.

In conclusion, this study confirmed the feasibility of generating accurate foot trajectories using the pendulum model of walking: foot trajectories at various speeds were accurately described by pendulum models. With the best-fit circle approximation algorithms, the ankle and heel trajectories at stance were well approximated by a single rigid pendulum. Furthermore, the toe trajectory over the whole gait cycle was well approximated by a pendulum. Investigation of the pendulum model in approximation of foot trajectories is a first step to employing the pendulum concept in the design of walking robots and lower limb orthoses.

Acknowledgments The first author was the recipient of a scholarship from the China Scholarship Council for PhD study in the School of Engineering, University of Glasgow [8].

\section{References}

1. Alexander RMcN (1976) Mechanics of bipedal locomotion. In: Davies PS (ed) Perspectives in experimental biology. Pergamon, Oxford, pp 493-504

2. Alexander RM, Goldspink G (1977) Mechanics and energetics of animal locomotion. Halsted Press, New York

3. Anderson SO, Wisse M, Atkeson CG, Hodgins JK, Zeglin GJ, Moyer B (2005) Powered bipeds based on passive dynamic principles. 5th IEEE-RAS international conference on humanoid robots, $110-116$

4. Banala SK, Hun SK, Agrawal SK, Scholz JP (2009) Robot assisted gait training with active leg exoskeleton (ALEX). IEEE Trans Neural Syst Rehabil Eng 17:2-8 
5. Coope ID (1993) Circle fitting by linear and nonlinear least squares. J Optim Theory Appl 76:381-388

6. Emken JL, Harkema SJ, Beres-Jones JA, Ferreira CK, Reinkensmeyer DJ (2008) Feasibility of manual teach-and-replay and continuous impedance shaping for robotic locomotor training following spinal cord injury. IEEE Trans Biomed Eng 55:322-334

7. Esquenazi A, Packel A (2012) Robotic-assisted gait training and restoration. Am J Phys Med Rehabil 91:S217-S231

8. Fang J (2013) Computer modelling and experimental design of a gait orthosis for early rehabilitation of walking. $\mathrm{PhD}$ Thesis, University of Glasgow, Glasgow

9. Fang J, Gollee H, Galen S, Allan DB, Conway BA, Vuckovic A (2011) Kinematic modelling of a robotic gait device for early rehabilitation of walking. Proc Inst Mech Eng $[\mathrm{H}]$ 225: $1177-1187$

10. Freivogel S, Schmalohr D, Mehrholz J (2009) Improved walking ability and reduced therapeutic stress with an electromechanical gait device. J Rehabil Med 41:734-739

11. Gard SA, Childress DS (2001) What determines the vertical displacement of the body during normal walking? J Prosthet Orthot 13:64-67

12. Hansen AH, Childress DS, Knox EH (2004) Roll-over shapes of human locomotor systems: effects of walking speed. Clin Biomech 19:407-414

13. Harkema SJ (2001) Neural plasticity after human spinal cord injury: application of locomotor training to the rehabilitation of walking. Neurosci 7:455-468
14. Hesse S, Waldner A, Tomelleri C (2010) Innovative gait robot for the repetitive practice of floor walking and stair climbing up and down in stroke patients. J NeuroEng Rehabil 7:30

15. Kadaba MP, Ramakrishnan HK, Wootten ME (1990) Measurement of lower extremity kinematics during level walking. J Orthop Res 8:383-392

16. Kasa I (1976) A circle fitting procedure and its error analysis. IEEE Trans Instrum Meas 25:8-14

17. Kuo AD (1999) Stabilization of lateral motion in passive dynamic walking. Int J Robot Res 18:917-930

18. Kuo AD, Donelan JM (2010) Dynamic principles of gait and their clinical implications. Phys Ther 90:157-174

19. Kwan M, Hubbard M (2007) Optimal foot shape for a passive dynamic biped. J Theor Biol 248:331-339

20. McGeer T (1990) Passive dynamic walking. Int J Robot Res 9:62-82

21. Mochon SM, McMahon TA (1980) Ballistic walking. J Biomech 13:49-57

22. Perry J (1992) Gait analysis: normal and pathological function. SLACK Incorporated, New Jersey

23. Winter DA (1992) Foot trajectory in human gait: a precise and multifactorial motor control task. Phys Ther 72:45-53

24. Winter DA (2005) Biomechanics and motor control of human movement, 3rd edn. Wiley, New York

25. Wisse M (2004) Three additions to passive dynamic walking; actuation, an upper body, and 3D stability. 4th IEEE/RAS international conference on humanoid robots 111: 113-132 